\title{
STREets, MALls, AND SUPERMARKETS
}

\author{
HOWARD SMITH \\ Department of Economics \\ University of Oxford \\ Oxford, UK OX1 3UQ \\ howard.smith@economics.ox.ac.uk \\ DONALD HAY \\ Department of Economics \\ University of Oxford \\ Oxford, UK OX1 3UQ
}

We develop a model of competition between shopping centers, comparing competitive outcomes in three alternative modes of retail organization, namely: streets (in which neither developers or retailers internalize agglomeration effects between products); malls (in which developers internalize); and supermarkets (in which both developers and retailers internalize). For a fixed number of centers: (i) converting streets to malls intensifies developer (but not retailer) competition, which increases product range (i.e., the number of shops built by the developers) and consumer surplus, reduces profits, and has ambiguous effects on welfare; (ii) converting streets to supermarkets intensifies retailer and developer competition, has ambiguous effects on product range (number of shops), reduces profits, and increases social welfare. With free entry both conversions reduce the number of centers and, if there is excess entry, conversion to supermarkets (but not malls) unambiguously increases welfare.

\section{INTRODUCTION}

Two features are present in many retail markets. ${ }^{1}$ First, consumers prefer one-stop shopping, which introduces agglomeration effects between products at any shopping center. Second, retailers enjoy positive pricecost margins, because of fixed costs and spatial differentiation between centers. The combination of these creates a potential for inefficiency, and

We are very grateful to the editor, the subeditor, and two anonymous referees, for helpful comments on an earlier draft, which greatly improved the paper. We are also grateful for useful comments from Konrad Stahl.

1. See Stahl (1987) for a survey of the economic characteristics of retailing.

(C) 2005 Blackwell Publishing, 350 Main Street, Malden, MA 02148, USA, and 9600 Garsington Road, Oxford OX4 2DQ, UK.

Journal of Economics \& Management Strategy, Volume 14, Number 1, Spring 2005, 29-59 
invites internalization of the agglomeration effects between products at any center.

Internalization may occur at the level of the retailer setting the prices and quality levels, or the developer building the outlets and thereby choosing the number of retailers. There are three commonly observed ways of organizing shopping centers, each with a different extent of internalization. In shopping streets there are many independent developers and retailers, in malls there is one developer but many independent retailers, and in supermarkets there is one developer and one retailer. In advanced economies there has been a trend toward greater internalization: large supermarkets today sell distinct items that previously were sold separately by specialist retailers, e.g., meat, bread, clothes, books, medicines, gasoline, and financial services. In some countries public policy restricts the number and size of developments, and attempts to protect street retailing, thereby limiting the extent of internalization.

This paper develops a model of competition between shopping centers and studies the effect of internalization on prices, quality levels, the number of product lines, the number of shopping centers, and social welfare. To abstract from the effect of internalization on competition within a center, we assume product lines at any center are independent in demand, for any given number of customers going to the center. Thus, cross-effects between product lines are brought about entirely through a change in the number of customers drawn to the center. For simplicity, we assume that product lines are symmetric, with identical cost and demand primitives. We assume that all consumers are identical up to location, are fully informed, demand all product lines, and visit a single center. We are motivated by the example of regular household shopping for nondurables where product lines are distinct demand categories that specialist retailers would sell in a street, as in the examples given above (meat, bread, clothes, etc.). To abstract from location choice, we assume that center locations are symmetric; consequently, centers compete with each other only through the consumer surplus $v$ offered to consumers ( $v$ is consumer surplus before transport costs). ${ }^{2}$

Competition is modeled as a three-stage game. In the first stage developers choose whether to enter-determining the number of centers, $K$. In the second, developers choose the product range, i.e., the number of product lines, $I$. This is a decision for developers because it requires investment in center size. In the third stage, retailers choose the utility $u$ to offer on each product line. The utility $u$ on any line is determined by

2. Other papers in which competition between "centers" is reduced to a choice of $v$ include Bliss (1988), Armstrong and Vickers (2001), and Armstrong (2004). 
the retailer's line-specific choice of price and quality. Quality is a product characteristic that increases consumer willingness to pay and is obtained by incurring a line-specific fixed cost, e.g., advertising, selection of merchandise, or sales assistance. The consumer surplus $v$ from a center is the sum of the utility $u$ on each of the $I$ product lines. By symmetry firms set a uniform level of $u$ for all I products. Developers charge each retailer a fixed rent, which fully extracts retailer profits, so there are no vertical externalities between the retailers and developers at a center.

We study three modes of center organization: (i) centers with a separate developer and retailer per product line so there is no internalization; (ii) centers with a single developer and a separate retailer per product line so there is developer internalization; and (iii) centers with a single developer and a single retailer so there is both developer and retailer internalization. We label these street, mall, and supermarket mode, respectively. We limit our attention to symmetric equilibria in which all centers are a given mode, and compare firm strategy and social welfare for market equilibrium for each mode.

We abstract from the effect of mode on cost and utility primitives, so the effect of internalization is entirely through firm conduct. ${ }^{3}$ When selecting $u$ and $I$ firms consider two effects on profits: a direct effect from existing customers and a market share effect from new customers. Internalization extends the market share effect to all products at the center, causing firms to attach extra importance to the utility $u$ they offer consumers.

To focus initially on retailer internalization, we fix $I$ and $K$ and change all centers from streets to supermarkets. This extends the retailer's market share effect to all products at the center. The retailer cannot discriminate, and offers a higher $u$ to all consumers, tolerating a negative direct effect in return for the market share effect. This is an intensification of competition, which implies lower profits from each product line in symmetric equilibrium, and thus lower center profits.

When $I$ is endogenous, developers enter the game. A change from streets to supermarkets now implies internalization by retailers and developers. The change extends the developer's market share effect (from adding a candidate product line) to all existing products at the center. But this incentive to increase $I$ is counteracted by retailer internalization, which intensifies stage 3 competition and reduces direct profit from each product line, including the candidate product line. There are thus two opposing effects and product range may rise or fall; in either case we find

3. As a referee points out, in practice some types of consumers may prefer certain modes of shopping, while malls and streets may have higher developer costs than supermarkets. 
that center profits fall in symmetric equilibrium because competition between centers has intensified.

A change from streets to malls introduces developer internalization without retailer internalization. This increases the developer's market share effect without reducing the direct profit on any line, so that $I$ unambiguously increases. This is an intensification of product line competition, and center profits fall in symmetric equilibrium.

The number of centers $K$ is determined in free-entry equilibrium. A change from streets to supermarkets (or malls) reduces center profit for any given $K$; this reduces the number of centers $K$ in free-entry equilibrium.

The social benefit of internalization is determined by the rate at which firms are willing to give up profit on existing customers to gain market share. Because all consumers visit a center, and centers are symmetric, the extra profit from new consumers, i.e., the market share effect, is offset exactly by the profit lost at other centers (a negative externality) with no overall effect on welfare. Thus, internalization increases welfare only if the extra utility offered exceeds the profit firms are willing to give up on existing consumers. ${ }^{4}$

In the simple case where $I$ and $K$ are fixed, a change of mode from streets to supermarkets increases the profit retailers are willing to sacrifice in return for new consumers. The effect on welfare is positive, however, since retailers can offer the utility efficiently from a social point of view-i.e., each dollar of profit forgone by retailers always increases consumer surplus by at least a dollar-and because retailers always have the option of offering the utility through a price cut. ${ }^{5}$

When $K$ is fixed but $I$ is endogenous, the developer's choice of $I$ enters the story. After a change from streets to supermarkets the developer is willing to add product lines that are not directly profitable, in return for new shoppers on all product lines. (Again, this market share effect is offset one-for-one by losses at other centers and thus does not contribute to social welfare.) Because the developer's profits are appropriated entirely from the retailer, the developer is not willing to sacrifice more profit than the retailer would, in return for any given number of new shoppers. Therefore, since we know that the retailer

4. This is identical to the condition that the consumer surplus effect exceeds the business stealing effect, because firms equate the profit sacrifice on existing customers to the business stolen from other centers.

5. The discussion is closely related to Spence (1975) where social inefficiency in quality and pricing is because firms consider the effect on marginal rather than average consumer surplus. In our model retailers attach some importance to average consumer surplus, because they care about the market share effect. Internalization increases the importance retailers give to average consumer surplus, $(v)$, i.e., they internalize some of the consumer benefit. 
gives up less than the consumers' gain (from the previous paragraph), it follows that the developer also gives up less than the consumers' gain, so the welfare change of going from street to supermarket mode is positive. In contrast, a change from streets to malls has more ambiguous welfare consequences. As before, the conversion increases the profit the developer is willing to sacrifice in return for new shoppers. But now each retailer is limited only to a single product, while the developer appropriates the market share effect of I products; consequently, the developer is prepared to sacrifice $I$ times as much as any retailer in return for a given market share change. It is now possible that the developer is willing to sacrifice more profit than is socially efficient, resulting in an over-supply of product lines and a fall in welfare relative to streets. ${ }^{6}$

Now suppose $K$ is endogenous, determined by free entry. If there is excess entry, the typical case in spatial models, ${ }^{7}$ then the $K$-reducing effect of a change from streets to supermarkets increases welfare, adding to the welfare gains just noted for fixed $K$. (In contrast, since conversion to malls has an ambiguous welfare effect for fixed $K$, this ambiguity remains with free entry.)

Two related papers compare centers where internalization is present (monopolist) and absent (specialist retailers). In contrast to our model, which focuses on competition between centers, these models hold prices at rival centers fixed and focus on behaviour within centers. In Stahl (1982), products are intrinsic substitutes, i.e., before agglomeration effects, but monopolists nonetheless charge lower prices than specialized retailers, by internalization of agglomeration effects. Schulz and Stahl (1996) use a search-theoretic setting where imperfectly informed consumers each buy one product. The number of products offered increases the attractiveness of a center, by increasing the chance of a good match between consumer and product. Because of internalization, the monopolist offers more products, and, for each number of products, charges lower prices. Overall, internalization increases welfare.

Two further papers consider competition between centers. Beggs (1992) endogenizes the internalization decision (which our model takes as given), showing that it may be a perfect Nash equilibrium for developers not to internalize at retail level-e.g., to build malls rather than supermarkets. Gehrig (1998) endogenizes the decision of retailers to agglomerate, i.e., to locate together at given locations (which our

6. The results on product lines are similar to those in Spence (1976): the addition of a product line depends on the proportion of the consumer surplus that is captured. With retailer internalization prices are lower, less of the consumer gain is internalized, and too few lines are offered. Without retailer internalization, prices are higher, more consumer surplus if internalized, and too many product lines may be offered.

7. See Anderson et al. (1992) and Mankiw and Whinston (1986). 
model takes as given). Retailers choose two horizontal characteristics, one of which is location, with agglomeration effects that retailers do not internalize. Consumers visit a single center, buying only one variant, and are not informed about the variants' characteristics before visiting. Consumers prefer centers with more retailers, and a better chance of successful search. Whether there are multiple market places, as opposed to complete agglomeration, depends on market size and transportation costs.

The search-theoretic setting of Gehrig (1998), and Schulz and Stahl (1996), is applicable to specialized, infrequent purchases of durable products where consumers are not well informed initially, and only buy one product at the end of the process. Our model, in contrast, is applicable to situations where consumers are fully informed, perhaps through experience, about the offerings at each center, buy a range of products on a shopping trip, and care about vertical product characteristics. A natural application is regular household shopping for nondurables such as groceries, clothing, medicines, financial services, etc.

Gould et al. (2002) and Pashigian et al. (1998) present empirical evidence from shopping malls that demonstrates the empirical importance of externalities between product lines, and show that owners of malls are willing to reduce rent for stores that generate positive externalities for other traders at the mall.

In a broader sense, the paper is related to the literature on intermediation, because the developer may be seen as an intermediary between consumers and retailers. For a discussion see Armstrong (2004), Nocke et al. (2004), and, more generally, Spulber (1999).

The rest of the paper is as follows. Section 2 presents the model's assumptions. Section 3 examines retail competition, holding the number of centers and product lines fixed. Sections 4 and 5 analyze developer decisions, namely number of product lines and entry. Section 6 briefly considers whether a developer would like to change mode. Section 7 discusses social efficiency. Section 8 concludes.

\section{A MODEL OF SHOPPING CENTERS}

We take as a given that all consumers visit a center and shopping costs prohibit any consumer visiting more than one. A consumer values center $k$ according to

$w_{k}=q_{0}+v_{k}-\varepsilon_{k}$

where $q_{0}$ is a numeraire good representing the rest of the economy, $v_{k}$ is utility from center $k$ and $\varepsilon_{k}$ is the consumer's transportation cost. Each component of $w_{k}$ is a continuous scalar. From the set of $K$ 
centers the consumer selects the one with the highest $w_{k}$. The good $q_{0}$ absorbs income effects so $v_{k}$ equals consumer surplus from $k$ (up to transportation costs). All consumers have identical $v_{k}$ for each $k$ so the only consumer variation is in transportation costs.

Consumer and center locations are exogenous, so competition between centers is entirely through the vector of utilities $\mathbf{v}=\left(v_{1}, \ldots, v_{K}\right)$. For any $\mathbf{v}$ there is a unique vector of market shares $\mathbf{s}=\left(s_{1}, \ldots, s_{K}\right)$. The market share $s_{k}$ of center $k$ is given by the function $s_{k}=s_{k}(\mathbf{v})$, which is symmetric in $\mathbf{v}$ such that equal center utilities $\left(v_{1}=v_{2}=\cdots=v_{K}\right)$ imply equal center shares $\left(s_{1}=s_{2}=\cdots=s_{K}\right)$. We require that $s_{k}()$ is continuous and twice differentiable in $\mathbf{v}$ such that ${ }^{8}$

$\frac{\partial s_{k}}{\partial v_{l}} \begin{cases}>0 & \text { for } k=l \\ \leq 0 & \text { for } k \neq l,\end{cases}$

where the own-utility derivative $(k=l)$ depends on $s_{k}$ or $v_{k}$ or both (and not on any other variable, except through $s_{k}$ or $v_{k}$ ). We do not impose any single distribution of transport costs, but note that the uniform distribution (which gives the "circular-city" Salop model) and the Type- 1 extreme value distribution (which gives the logit oligopoly model) satisfy these requirements (and we use them in examples). ${ }^{9}$

Each center offers $I_{k}$ product lines, which are independent in demand, with $v_{k}$ additively separable in the $I_{k}$ product utilities, i.e., $v_{k}=u_{1 k}+\cdots+u_{I_{k} k}$. The indirect utility function for product $i$, written as $u_{i k}=u\left(p_{i k}, n_{i k}\right)$, is decreasing in price $p_{i k}$ and increasing in quality $n_{i k}$ and implies product demand $q_{i k}=q\left(p_{i k}, n_{i k}\right)=-\partial u_{i k} / \partial p_{i k}$ that is nonincreasing in $p_{i k}$ and nondecreasing in $n_{i k}$. Functions $\{u(), q()\}$ are identical for all consumers. ${ }^{10}$

There are two types of firms, developers and retailers. Developers choose whether to open a center $k$ and decide on the number of lines $I_{k}$ to offer, incurring a fixed cost $f\left(I_{k}\right)$ that is increasing in $I_{k}$. Retailers set price $p_{i k}$ and quality $n_{i k}$ and obtain retailer profit $\pi_{i k}$ on each product. No developer or retailer operates in more than one center. Developers

8. Continuity of $s_{k}()$ in $\mathbf{v}$ ensures smooth profit functions in $\mathbf{v}$, needed to ensure existence of symmetric equilibria. As noted by a referee, there are models of vertical differentiation (e.g., Shaked and Sutton, 1982) where symmetric equilibria do not exist, as $s_{k}()$ is discontinuous if firms set identical $v$, giving any firm an incentive to deviate, e.g., by increasing quality. The presence of transportation $\operatorname{costs} \varepsilon_{k}$ allows $s_{k}()$ to be continuous in $\mathbf{v}$.

9. In the logit model $\frac{\partial s_{k}}{\partial v_{k}}=s_{k}\left(1-s_{k}\right) / t$ and is unaffected by $s_{l}$ except through $s_{k}$ (where $t$ is the scale parameter of the distribution). In the Salop model $\frac{\partial s_{k}}{\partial v_{k}}=1 / t$ so it is independent of $s_{k}$ as well as independent of $s_{l}$. See Anderson et al. (1992). It is known that the Salop model has discontinuities at values of $v$ outside the relevant range.

10. It should be pointed out that independent demand functions is a strong assumption, employed to focus on the consequences of internalization for intercenter competition. 
have full information about the conditions facing retailers, and charge each retailer a fixed rent that extracts all retailer profit.

There are three modes $m$ of center organization, namely streets, malls, and supermarkets, i.e., $m \in\{s t, m a, s u\}$. Mode is exogenous and common across the $K$ centers. The modes are organized as follows. In a street with $I_{k}$ lines there are $I_{k}$ developers and $I_{k}$ retailers, in a mall there is one developer and $I_{k}$ retailers, and in a supermarket there is one developer and one retailer. Retailers in streets or malls are single-category shops (butchers, bakers, clothes stores, etc). In street mode each shop is developed independently; in mall mode they are all developed by the same developer. Each mode implies a different pattern of internalization of a center's agglomeration effects. In street mode there is none, either by retailers or developers, in mall mode the developer internalizes, and in supermarket mode developers and retailers internalize. To abstract from competition within a center, a given product line is never offered twice in the same center. To abstract from other effects of mode, cost and utility primitives are unaffected by mode.

There are three decision stages. First, developers decide whether to build a center. (In street mode we assume that there are no coordination problems between retailers at this stage.) Second, developers select the number of product lines $I_{k}$. Third, retailers set price $p_{i k}$ and quality $n_{i k}$ for each product.

In stage 3 we take as given the number of centers $K$ and a product range $I_{k}$ at center $k$. The retailer of product $i$ at center $k$ sets price $p_{i k}$, and can increase quality $n_{i k}$ above the minimum level $n_{i k}=0$ by incurring a fixed cost $g_{i k}=g\left(n_{i k}\right)$, where $g^{\prime}()>0$. Marginal cost $c_{i k}$ is constant and unaffected by quality. (Natural examples of quality are staff helpfulness, store layout, and good merchandise selection.) Product costs $c_{i k}$ and $g_{i k}$ are unaffected by the quality or output of products other than $i$. For symmetry, we specify that the primitives of the retailer game $\{u(), q()$, $c(), g()\}$ are identical for all $i$ and $k$.

There are no cost or demand cross-effects between products at center $k$, up to market share $s_{k}$. It follows that internalization has no effect on retailer choice of $p_{i k}$ and $n_{i k}$ except through $u_{i k}$ and $s_{k}$. Accordingly, we can write product $i^{\prime}$ s retail profit $\pi_{i k}$ in terms of $u_{i k}$ and $s_{k}$, with $p_{i k}$ and $n_{i k}$ implicitly set at profit-maximizing levels:

$\pi_{i k}=\pi\left(u_{i k}, s_{k}(\mathbf{v})\right) \equiv \max _{p_{i k} n_{i k}}\left\{s_{k} q_{i k}\left(p_{i k}-c_{i k}\right)-g_{i k} \mid u_{i k}=u\left(p_{i k}, n_{i k}\right)\right\}$

where we assume $\pi()$ is continuous and differentiable in both arguments. The problem of the retailer of $i$ at center $k$ is therefore reduced to utility-setting, as follows: 
$\begin{array}{ll}\max _{u_{i k}} \pi\left(u_{i k}, s_{k}(\mathbf{v})\right) & \text { if mall or street mode } \\ \max _{\mathbf{u}_{k}} \sum_{i=1}^{I_{k}} \pi\left(u_{i k}, s_{k}(\mathbf{v})\right) & \text { if supermarket mode. }\end{array}$

The function $\pi()$ is identical for all $i$ and $k$, by symmetry of $\{u(), q()$, $c(), g()\}$. To ensure each retailer has a unique profit-maximizing utility, the objective function is assumed strictly quasiconcave (in $u_{i k}$ for streets or malls and in $\mathbf{u}_{k}$ for supermarkets). By symmetry, profit-maximizing retailers set $u_{i k}$ uniformly across the products at any center.

The derivative of the objective function (3) with respect to $u_{i k}$ on $i$ is

$\rho^{m}\left(u_{i k}, s_{k} \mid I_{k}\right) \equiv \rho_{i k}^{m}=\frac{\partial \pi\left(u_{i k}, s_{k}\right)}{\partial u_{i k}}+x^{m} \frac{\partial \pi\left(u_{i k}, s_{k}\right)}{\partial s_{k}} \frac{d s_{k}}{d u_{i k}}$,

where $x^{m}=1$ in streets or malls and $x^{m}=I_{k}$ in supermarkets. (The latter follows because the center's $I_{k}$ products are set to a uniform utility, so that by symmetry an increase in $s_{k}$ has an identical effect on the profits of all products at center $k$.) The first term on the right-hand side is the direct effect of the product's utility $u_{i k}$ on product $i$ 's profits, holding $s_{k}$ constant. The second term is the market share effect derived from all the retailer's $x^{m}$ product lines. In street mode the retailer's market share effect is limited to product $i$ 's profit. In supermarket mode it is enjoyed on all $I_{k}$ products at the center. It is standard in oligopoly that $\pi()$ gives a negative direct effect for high enough levels of $u_{i k}$. (Otherwise there would be no interior solution in $u_{i k}$ for profit maximization with fixed market share, e.g., for a monopolist or a group of colluding oligopolists.) The market share effect is always positive, as products always have positive markups in equilibrium. ${ }^{11}$

To ensure the existence of unique symmetric equilibrium, we restrict $\rho()$ such that, for each mode $m$ and any $s_{k}$ in $0<s_{k} \leq 1$, there is always a unique finite utility $\hat{u}_{k}^{m}=\hat{u}^{m}\left(s_{k} \mid I_{k}\right)$ above which $\rho_{k}^{m}<0$ and below which $\rho_{k}^{m}>0$. That is,

$\rho^{m}\left(u_{i k}, s_{k} \mid I_{k}\right) \begin{cases}<0 & \text { for } u_{i k}>\hat{u}^{m}\left(s_{k} \mid I_{k}\right) \\ >0 & \text { for } u_{i k}<\hat{u}^{m}\left(s_{k} \mid I_{k}\right)\end{cases}$

for each mode $m$ and any $s_{k}$ in $0<s_{k} \leq 1$. At any market share, the first line in (5) requires that, for high enough $u_{i k}$, the direct effect eventually becomes sufficiently negative to outweigh the market share

11. Agglomeration effects may, in some circumstances, result in negative markups on some product lines (see Bliss, 1988). However, where products are symmetric, and independent in demand, as in our application, markups are identical and positive. 
effect. This is natural since, as $u_{i k}$ increases, $p_{i k}-c_{i k}$ eventually goes to zero or $g\left(n_{i k}\right)$ becomes large. The second line requires that at any $s_{k}$, reducing $u_{i k}$ is eventually unprofitable, which is natural since the demand $q()$ per customer goes to zero at low enough $u_{i k}$. (For example, at the collusive $u_{i k}$ it is profitable for any retailer to increase utility.) Given that $\pi_{k}$ is continuous and differentiable in $u_{k}$ and $s_{k}$, condition (5) implies $\rho\left(\hat{u}_{k}^{m}, s_{k} \mid I_{k}\right)=0$. As the following proposition shows, there is a unique symmetric equilibrium level of utility for each mode $m$, for any given $K$ and common $I_{k}$ across centers, which we denote as $\hat{u}_{K}^{m}=\hat{u}_{K}^{m}\left(I_{k}\right)$. (Throughout the paper, a $K$ subscript denotes a symmetric equilibrium value with $K$ centers.)

Proposition 1: A unique symmetric equilibrium in product utility $\hat{u}_{K}^{m}=$ $\hat{u}_{K}^{m}\left(I_{k}\right)$ exists for each mode $m$ and any number of centers $K$ with common $I_{k}$ at each center.

Proof. For each mode $m$, and any $s_{k}$ in the range $0<s_{k} \leq 1$, condition (5) implies a unique finite utility $\hat{u}_{i k}^{m}=\hat{u}^{m}\left(I_{k}\right)$ satisfying $\rho_{i k}^{m}=0$ for product $i$ at center $k$ with $I_{k}$ lines. By strict quasiconcavity of each retailer's profit function in own utility $u_{i k}$ this is a unique best reply, and is identical for all $i$ at center $k$. Let $\hat{u}_{K}^{m}=\hat{u}_{K}^{m}\left(I_{k}\right)$ denote the unique best reply, for all $i$ at center $k$, satisfying $\rho^{m}\left(\hat{u}_{K}^{m}, s_{k} \mid I_{k}\right)=0$ at $s_{k}=1 / K$. Suppose all retailers at centers other than $k$ set $\hat{u}_{K}^{m}$. If retailers at $k$ set $\hat{u}_{K}^{m}$ then, since all centers have $I_{k}$ products, they have the same center utility $v_{k}=I_{k} \hat{u}_{K}^{m}$, and the symmetry property of $s_{k}()$ implies $s_{k}=1 / K$. Then $\hat{u}_{K}^{m}$ is the unique best reply for $k$ and all centers are in unique symmetric equilibrium.

Because this proposition shows that retailers at any center $k$ set a uniform utility on all products, we hereafter drop $i$ subscripts, i.e., $u_{k}=u_{i k}$ for all $i$.

In stages 1 and 2, developers decide whether to enter and how many product lines to offer. The total fixed cost of a center with a capacity for $I_{k}$ product lines is given by the function $f\left(I_{k}\right)$, which is continuous and differentiable in $I_{k}$ such that the fixed cost of an extra product line is positive and increasing, i.e., $\left\{f^{\prime}(), f^{\prime \prime}()\right\}>0$. This ensures an interior solution in the number of product lines. (We ignore the integer constraint to simplify notation.) Let $\Pi_{k}$ be aggregate developer profit at center $k$, comprising total retail profit minus total developer cost $f\left(I_{k}\right)$, i.e.,

$\Pi\left(I_{k}, \hat{u}_{k}^{m}, s_{k}\right)=\Pi_{k}=I_{k} \pi\left(\hat{u}_{k}^{m}, s_{k}\right)-f\left(I_{k}\right)$

where retailers at $k$ set $\hat{u}_{k}^{m}=\hat{u}^{m}\left(s_{k} \mid I_{k}\right)$ on all products in stage 3 . (In street mode $\Pi_{k}$ is the aggregation of the profits of the individual developers 
at center $k$; in the other modes it is the profit of the sole developer at center $k$.)

In supermarkets or malls, a single developer sets $I_{k}$ to maximize aggregate developer profit $\Pi_{k}$. In a street, by contrast, there is a developer for each line, offering the line if it is profitable in terms of its own revenues and costs, i.e., not considering effects on other products at $k$. Marginal developer profit $\delta_{k}^{m}$ from an extra product line in center $k$ of mode $m$ is obtained by differentiating the relevant objective function for mode $m$ with respect to $I_{k}$, giving

$\delta^{m}\left(I_{k}, s_{k}\right) \equiv \delta_{k}^{m}=\pi_{k}-f^{\prime}\left(I_{k}\right)+y^{m} \frac{\partial \pi_{k}}{\partial s_{k}} \frac{d s_{k}}{d v_{k}} \hat{u}_{k}^{m}$,

where $y^{m}=1$ in street mode and $y=I_{k}$ in mall and supermarket mode. Marginal developer profit, like marginal retailer profit, is made up of a direct effect, the first two terms, and a market share effect, the final term. In the final term, $\hat{u}_{k}^{m}$ is the effect on consumer surplus $v_{k}$ of a marginal increase in $I_{k}$.

As $I_{k}$ increases, $f^{\prime}()$ increases, and the direct effect falls, becoming negative for large enough $I_{k}$. The market share effect is always positive (as profit margins are always positive). At some point, however, the direct effect becomes sufficiently negative to outweigh the market share effect, so that $\delta_{k}^{m}=0$; developers add lines until this point is reached. To ensure equilibrium, we assume the marginal developer profit satisfies the following quasiconcavity property: $\delta^{m}$ is initially positive up to some $I_{k}$ and is negative thereafter, given any vector of product lines $\mathbf{I}_{-k}$ at rival centers, with $\hat{u}_{k}^{m}=\hat{u}^{m}\left(s_{k} \mid I_{k}\right)$ determined by retailer competition in stage 3.

To ensure the existence of unique symmetric equilibrium in product lines we specify that, for any mode $m$, and market share $s_{k}$ in the range $0<s_{k} \leq 1$, there is always some unique number of product lines $\hat{I}^{m}\left(s_{k}\right)$ above which marginal developer profit is negative, and below which marginal developer profit is positive, i.e.,

$\delta^{m}\left(I_{k}, s_{k}\right) \begin{cases}<0 & \text { for } I_{k}>\hat{I}^{m}\left(s_{k}\right) \\ >0 & \text { for } I_{k}<\hat{I}^{m}\left(s_{k}\right) .\end{cases}$

This condition is reasonable, since it is natural that, for any market share, a developer should (i) find it worthwhile to add a marginal product at low enough $I_{k}$ and (ii) find that profit opportunities are exhausted at some $I_{k}$ high enough. The condition requires that $f^{\prime}()$ is sufficiently small at low $I_{k}$ and sufficiently large at high $I_{k}$. Since $\delta^{m}()$ is continuous and differentiable, condition (7) implies that $\delta^{m}=0$ at $\hat{I}_{k}^{m}$. As Proposition 2 
shows, these conditions imply the existence of a unique symmetric equilibrium product range, for each $m$ and any $K$, denoted $\hat{I}_{K}^{m}$.

Proposition 2: A unique symmetric perfect equilibrium exists in product lines $\hat{I}_{K}^{m}$ for each $m$ and any $K$.

Proof. For each mode $m$ and any $s_{k}$ in $0<s_{k} \leq 1$, condition (7) implies there is a unique finite $\hat{I}_{k}^{m}=\hat{I}^{m}\left(s_{k}\right)$ satisfying $\delta_{k}^{m}=0$ for a representative developer at $k$ assuming that optimal utility, $\hat{u}_{k}^{m}=\hat{u}^{m}\left(s_{k} \mid \hat{I}_{k}^{m}\right)$, is set by retailers. By the quasiconcavity property, $\hat{I}_{k}^{m}$ is a unique best reply. Let $\hat{I}_{K}^{m}$ denote the unique best reply satisfying $\delta_{k}^{m}\left(\hat{I}_{K}^{m}, s_{k}\right)=0$ at $s_{k}=1 / K$. Suppose the number of product lines at centers other than $k$ is $\hat{I}_{K}^{m}$ and utility at these centers is $\hat{u}_{K}^{m}$. If the developer at $k$ sets $\hat{I}_{K}^{m}$ then its retailers set $\hat{u}_{K}^{m}$ and center utility $v_{k}$ is the same across centers, so the symmetry property of $s_{k}()$ gives $s_{k}=1 / K$. Then $\hat{I}_{K}^{m}$ is the unique best reply for the developer at $k$ and all centers are in a unique symmetric equilibrium.

\section{Utility, Price, ANd Quality}

In this section we study the effect of mode on retail competition. We take $I_{k}$ and $K$ as given, with $I_{k}>1$ at a common level for all centers. Streets and malls are identical when $I_{k}$ is taken as given, so in this section we refer only to streets. Initially we reduce intercenter competition to a choice of $u_{k}$, with $p_{k}$ and $n_{k}$ implicitly set to maximize $\pi_{k}$.

At center $k$ of mode $m$, consider a representative retailer with $x^{m}$ product lines. The derivative of the retailer's profit with respect to $u_{k}$ on a given product is the sum of the direct and market share effects:

$\rho^{m}\left(u_{k}, s_{k}\right) \equiv \rho_{k}^{m}=\frac{\partial \pi\left(u_{k}, s_{k}\right)}{\partial u_{k}}+x^{m} \frac{\partial \pi\left(u_{k}, s_{k}\right)}{\partial s_{k}} \frac{d s_{k}}{d u_{k}}$

as previously given in (4). A profit-maximizing retailer sets $\rho_{k}^{m}=0$. Symmetric equilibrium utility is given by the utility satisfying this condition at $s_{k}=1 / K$, written as $\hat{u}_{K}^{s t}$ and $\hat{u}_{K}^{s u}$ for street and supermarket mode, respectively. The implied symmetric product profits are written as $\hat{\pi}_{K}^{s t}$ and $\hat{\pi}_{K}^{s u}$.

Condition (8) shows the effect of mode on $\hat{u}_{K}^{m}$. Consider initially street equilibrium where retailers set $\hat{u}_{K}^{s t}$ satisfying $\rho^{s t}\left(\hat{u}_{K}^{s t}, 1 / K\right)=0$. A switch to supermarkets increases $x^{m}$ from 1 to $I_{k}$, extending the market share effect to all $I_{k}$ products. As the market share effect on each product line is positive, the marginal profit of $u_{k}$ increases and is now positive, i.e., $\rho^{s u}\left(\hat{u}_{K}^{s t}, 1 / K\right)>0$. Condition (5) requires that $u_{k}$ increases to satisfy $\rho^{s u}\left(u_{k}, 1 / K\right)=0$. Let $\hat{u}_{K}^{s u}$ be the required new level. If all centers set $\hat{u}_{K}^{s u}$ then market shares are equal at $s_{k}=1 / K$ and $\rho^{s u}\left(\hat{u}_{K}^{s u}, 1 / K\right)=0$ for all $k$. 
The change to supermarkets therefore increases $\hat{u}_{K}^{m}$ to a new symmetric equilibrium.

To see the effect of mode on equilibrium product profit $\hat{\pi}_{K}^{m}$ recall that the direct effect in (8) is negative at $\hat{u}_{K}^{m}$ and $s_{k}=1 / K$. Conversion to supermarkets increases $\hat{u}_{K}^{m}$ and leaves $s_{k}$ unchanged, so $\hat{\pi}_{K}^{m}$ falls. This is intuitive, as a retailer's motive to increase $u_{k}$ is derived only from market share effects, which cancel out in symmetric equilibrium.

Proposition 3 summarizes.

Proposition 3: In symmetric equilibrium with given $I_{k}$ and $K$ a change from streets to supermarkets implies: (i) higher product utility $\left(\hat{u}_{K}^{s u}>\hat{u}_{K}^{s t}\right)$ and (ii) lower product profits $\left(\hat{\pi}_{K}^{s u}<\hat{\pi}_{K}^{s t}\right)$.

Proof. (i) Let $\hat{u}_{K}^{s u}$ and $\hat{u}_{K}^{s t}$ denote the unique symmetric equilibrium utility solving $\rho^{m}\left(u_{k}, 1 / K\right)=0$ for $m=s u$ and $m=s t$, respectively, at any $K$ and $I_{k}$. The effect of $m$ on $\rho^{m}()$ is exclusively through $x^{m}$, which increases from 1 to $I_{k}$ when $m$ changes from st to $s u$. By strict positive monotonicity of $\pi_{k}()$ in $s_{k}$, and $s_{k}()$ in $u_{k}$, the second term in (8) is positive, so $\rho^{m}()$ is increasing in $x^{m}$. This gives $\rho_{k}^{s u}\left(\hat{u}_{K}^{s t}, 1 / K\right)>0$ and, by the second line in (5), the condition for equilibrium utility $\rho_{k}^{s u}\left(\hat{u}_{K}^{s u}, 1 / K\right)=0$ is satisfied only for $\hat{u}_{K}^{s u}>\hat{u}_{K}^{s t}$. (ii) Let $\hat{\pi}_{K}^{s t}$ denote product profit in symmetric street equilibrium with $K$ centers, i.e., $\hat{\pi}_{K}^{s t}=\pi\left(\hat{u}_{K}^{s t}, 1 / K\right)$. Consider a series of hypothetical marginal increments in $x^{m}$ (in equation (8)) from 1 to $I_{k}$ simultaneously at all centers, maintaining utility at the equilibrium level $\hat{u}_{K}^{m}$ that solves $\rho^{m}\left(u_{k}, 1 / K\right)=0$ for each $x^{m}$. The change in equilibrium product profit from the conversion is given by taking the integral over the marginal profit effects, holding market share constant, i.e.,

$\hat{\pi}_{K}^{s u}-\hat{\pi}_{K}^{s t}=\int_{1}^{I_{k}}\left\{\frac{\partial \hat{\pi}_{K}^{m}}{\partial u_{k}} \frac{\partial \hat{u}_{K}^{m}}{\partial x^{m}}\right\} d x^{m}$

where $\hat{u}_{K}^{m}$ is the equilibrium utility solving $\rho^{m}\left(u_{k}, 1 / K\right)=0$ for each $x^{m}$. From part (i) of the proof we know that the marginal profit of utility, $\rho_{k}^{m}\left(u_{k}, s_{k}\right)$, is increasing in $x^{m}$, so that $\partial \hat{u}_{K}^{m} / \partial x^{m}>0$ for each $x^{m}$. Since $\rho^{m}\left(\hat{u}_{K}^{m}, 1 / K\right)=0$ for each $x^{m}$, and we know from part (i) that the second term in (8) is positive, it follows that $\partial \hat{\pi}_{K}^{m} / \partial u_{k}<0$, for each $x^{m}$. Therefore, the integrand in (9) is negative at each $x^{m}$, and the integral is negative, giving $\hat{\pi}_{K}^{s u}<\hat{\pi}_{K}^{s t}$.

We have shown that the supermarket mode implies an increase in $u_{k}$. In the rest of this section we examine the effect of this on prices and quality levels ( $p_{k}$ and $n_{k}$ ).

Suppose first that $n_{k}$ is fixed. Then, given that $u\left(p_{k}, n_{k}\right)$ is decreasing in $p_{k}$ and increasing in $n_{k}$, it follows that $p_{k}$ falls in the supermarket mode. 
Alternatively, if $p_{k}$ is fixed then $n_{k}$ must increase in the supermarket mode.

What if neither $n_{k}$ or $p_{k}$ is fixed? We can rule out the possibility that the supermarket mode would result in increased $p_{k}$ and reduced $n_{k}$, which could only be associated with a reduced level of $u_{k}$. Three remaining outcomes are possible: reduced $p_{k}$ and increased $n_{k}$, increased $p_{k}$ and $n_{k}$, and reduced $n_{k}$ and $p_{k}$. To explore this further, it is useful to recall that we take the retailer's problem in two conceptual stages: in the first stageeve choose $\hat{u}_{k}$ and then, in the second stage, choose $\hat{p}_{k}$ and $\hat{n}_{k}$ given $\hat{u}_{k}$. Because any $\hat{u}_{k}$ implies a unique $s_{k}$ (given utility choices of other retailers), the second stage is a constrained optimization problem for fixed $\bar{s}_{k}$ and $\hat{u}_{k}$ i.e.,

$$
\left.\begin{array}{l}
\hat{p}\left(\hat{u}_{k}, \bar{s}_{k}\right) \\
\hat{n}\left(\hat{u}_{k}, \bar{s}_{k}\right)
\end{array}\right\} \equiv \arg \max _{p_{k}, n_{k}}\left\{\bar{s}_{k} q\left(n_{k}, p_{k}\right)\left(p_{k}-c_{k}\right)-g\left(n_{k}\right) \mid \hat{u}_{k}=u\left(p_{k}, n_{k}\right)\right\}
$$

as given in (2). Provided $\{u(), q(), g(), c()\}$ are differentiable in $n_{k}$ and $p_{k}$ and the solution is interior, the Lagrangian for this problem satisfies the familiar condition:

$$
\frac{\partial \hat{\pi}_{k}}{\partial p_{k}} / \frac{\partial \hat{u}_{k}}{\partial p_{k}}=\frac{\partial \hat{\pi}_{k}}{\partial n_{k}} / \frac{\partial \hat{u}_{k}}{\partial n_{k}}
$$

where $\hat{\pi}_{k}$ is product profit at $\hat{p}\left(\hat{u}_{k}, \bar{s}_{k}\right)$ and $\hat{n}\left(\hat{u}_{k}, \bar{s}_{k}\right)$. The condition requires that the marginal cost of utility $u_{k}$ through a price cut equals the marginal cost of $u_{k}$ through a quality increase, where marginal cost is in terms of the retailer's profit forgone assuming a fixed market share $s_{k}$. The effect of the supermarket mode on $p_{k}$ and $n_{k}$ therefore depends on each variable's marginal cost of $u_{k}$ to the retailer, which is determined by the retailer's demand and cost functions.

In Example 1 the supermarket mode may result in reduced $p_{k}$ and increased $n_{k}$, increased $p_{k}$ and $n_{k}$, or reduced $n_{k}$ and $p_{k}$, depending on the shape of the demand function. We will outline the intuition for these results informally. (The specification of the model is detailed at the end of the section and the results are worked out fully in Smith, 2004).

First consider Case A of the example. Here the retailer has unit demands $q()$ that are zero-elastic in $n_{k}$ and $p_{k}$, up to a (nonbinding) reservation price that is increasing in $n_{k}$. Because demand is zero-elastic, a cut in $p_{k}$ transfers surplus one-for-one from retailer to consumers, so the marginal cost of $u_{k}$ through $p_{k}$ is a constant. In contrast, an increase in $n_{k}$ delivers surplus to consumers at increasing marginal cost, since $g^{\prime}()>0$. In this case it is clearly best for the supermarket mode to offer higher $u_{k}$ by reducing $p_{k}$, leaving $n_{k}$ unchanged. 
In Case B demand $q()$ is now downward-sloping in $p_{k}$ (such that $n_{k}$ does not influence $q()$ on the portion prices are set) so that the marginal cost of $u_{k}$ through $p_{k}$ is now increasing as $p_{k}$ falls. (As before, the marginal cost of $u_{k}$ through $n_{k}$ is also increasing.) It is now best for the supermarket to offer extra $u_{k}$ through higher $n_{k}$ as well as cuts in $p_{k}$.

In the next two cases (Cases C and D) we allow quality $n_{k}$ to increase the optimal price $\hat{p}_{k}$, by enhancing the consumer's valuation of marginal output. Suppose demand is constant, at $q=1$, for prices up to $n_{k}$, and elastic for higher prices. Retailers in both modes find it worthwhile to increase prices to $n_{k}$ but no higher. (Thus $n_{k}$ is the consumer's valuation of marginal output.) Because of the kink, there is a "jump" in the marginal cost of providing $u_{k}$ through $p_{k}$, and it is best for the supermarket to offer extra $u_{k}$ by changing $n_{k}$ instead. Because this moves the kink, it means a one-for-one change in $\hat{p}_{k}$.

In Case $C$ an increase in utility $u_{k}$ requires an increase in $n_{k}$ because the extra social surplus from $n_{k}$ exceeds the extra revenue extracted from the increase in $\hat{p}_{k}$, i.e., there is a positive consumer externality from $n_{k}$. However, the converse is also possible. In Case D there is a reduction in $n_{k}$ because the reduction in social surplus is less than the reduction in revenue extracted from the fall in $\hat{p}_{k}$. The difference between cases $\mathrm{C}$ and D hinges on the precise effect of $n_{k}$ on consumer demand $q()$. If $n_{k}$ affects the average valuation of output more than the valuation of marginal output, conversion to supermarkets increases $n_{k}$ and $p_{k}$, while in the opposite case conversion reduces $n_{k}$ and $p_{k}$.

Proposition 4 summarizes.

Proposition 4: In symmetric equilibrium with given $I_{k}$ and $K$ a change from streets to supermarkets may result in: (i) lower $\hat{p}_{K}^{m}$ and higher $\hat{n}_{K}^{m}$; (ii) higher $\hat{p}_{K}^{m}$ and $\hat{n}_{K}^{m}$; or (iii) lower $\hat{p}_{K}^{m}$ and $\hat{n}_{K}^{m}$, but not (iv) higher $\hat{p}_{K}^{m}$ and lower $\hat{n}_{K}^{m}$.

Proof. Cases B, C, and D of Example 1 (details below) give outcomes (i)-(iii) respectively. Outcome (iv) is not possible because $\hat{u}_{K}^{s u}>\hat{u}_{K}^{s t}$ (Proposition 3 ) and $u\left(p_{k}, n_{k}\right)$ is strictly decreasing in $p_{k}$ and strictly increasing in $n_{k}$.

EXAMPLe 1 [Symmetric Salop Circular-City]: Let consumers be uniformly distributed in a circle city of unit circumference with $K$ evenly spaced shopping centers. Let transportations costs $t$ be linear, giving $\partial s_{k} / \partial v_{k}=1 / t$. Suppose retailer costs are as follows: $\left\{c_{k}=0, g_{k}=\gamma n_{k}^{2} / 2\right\}$. Each consumer's marginal value of output (inverse demand) $P\left(q_{k}, n_{k}\right)$ is specified as follows:

$P\left(q_{k}, n_{k}\right)= \begin{cases}n_{k}^{a}+\left(\alpha+\beta n_{k}\right)\left(1-q_{k}\right) & \text { for } q_{k} \leq 1 \\ n_{k}^{a}-(1 / \phi)\left(q_{k}-1\right) & \text { for } q_{k}>1 .\end{cases}$ 
In Case A we set $\{a=1, \alpha=\beta=\phi=0, t<1 / \gamma\}$, which implies $\hat{p}_{K}^{s u}<$ $\hat{p}_{K}^{s t}$ and $\hat{n}_{K}^{s u}=\hat{n}_{K}^{s t}$. In Case B we set $\{a=\alpha=0,(\phi>0, \beta>0\}$, which implies $\hat{p}_{K}^{s u}<\hat{p}_{K}^{s t}$ and $\hat{n}_{K}^{s u}>\hat{n}_{K}^{s t}$. In Case C we set $\{\phi=0, b=1, \alpha>$ $0, \beta>0\}$, giving $\hat{p}_{K}^{s u}>\hat{p}_{K}^{s t}$ and $\hat{n}_{K}^{s u}>\hat{n}_{K}^{s t}$. In Case $\mathbf{D}$ we set $\{\phi=0, b=$ $1, \alpha>0, \beta<0\}$, giving $\hat{p}_{K}^{s u}<\hat{p}_{K}^{s t}$ and $\hat{n}_{K}^{s u}<\hat{n}_{K}^{s t}$. See Smith (2004) for a derivation of these results.

\section{Product Range}

We now examine the effect of mode on product range $\hat{I}_{K}^{m}$, holding $K$ constant. We define $\hat{I}_{K}^{m}$ as a (symmetric perfect) equilibrium if it is a center's best reply when all other centers set $\hat{I}_{K}^{m}$. Two levels of internalization are now relevant: developer level, increasing the incentive to add a line, and retailer level, which reduces retail profit, diminishing the incentive to add a line. We compare all three modes: streets (no internalization), malls (developer internalization only), and supermarkets (retailer and developer internalization).

At center $k$ of mode $m$ consider a developer with $y^{m}$ product lines. In malls and supermarkets the developer operates all lines at the center (so that $y^{m a}=y^{s u}=I_{k}$ ). In streets a developer only operates a single line (so that $\left.y^{s t}=1\right)$. A developer's marginal profit $\delta_{k}^{m}$ from the Ith product line is given by

$\delta^{m}\left(I_{k}, s_{k}\right) \equiv \delta_{k}^{m}=\pi\left(\hat{u}_{k}^{m}, s_{k}\right)-f^{\prime}\left(I_{k}\right)+y^{m} \frac{\partial \pi\left(\hat{u}_{k}^{m}, s_{k}\right)}{\partial s_{k}} \frac{d s_{k}}{d v_{k}} \hat{u}_{k}^{m}$

as given previously in (6). The first two terms are the direct effect and the final term is the market share effect. Product utility $\hat{u}_{k}^{m}$ is determined in stage 3 , given $I_{k}$. In mall and supermarket mode a single developer chooses $I_{k}$ to set $\delta_{k}^{m a}=0$ or $\delta_{k}^{s u}=0$, maximizing center profit. In the street mode single-product developers enter until $\delta_{k}^{s t}=0$, when individualproduct profit opportunities are exhausted, and in this mode $I_{k}$ equals the number of developers.

The first-order condition in equation (12) shows the effect of mode on $\hat{I}_{K}^{m}$. We make three comparisons: streets with malls, streets with supermarkets, and malls with supermarkets. First, suppose all centers are initially streets, setting $\hat{I}_{K}^{s t}$ so that $\delta^{s t}\left(\hat{I}_{K}^{s t}, 1 / K\right)=0$, and consider a change to malls. This increases $y^{m}$ from 1 to $I_{k}$ extending the developer's market share effect to all products. Retailer behavior is unaffected, so there is no change in $\hat{\pi}_{K}^{m}$, and therefore the direct effect is unchanged. There is also no change anything other than $y^{m}$ in the market share effect, which therefore increases in proportion to the change in $y^{m}$. This increases $\delta_{k}^{m}$, giving $\delta^{m a}\left(\hat{I}_{K}^{s t}, 1 / K\right)>0$. Condition (7) says that $I_{k}$ must 
increase to satisfy $\delta^{m a}\left(I_{k}, 1 / K\right)=0$. Let $\hat{I}_{K}^{m a}$ denote the required higher level. If all centers set $\hat{I}_{K}^{m a}$ market shares are equal at $s_{k}=1 / K$ and $\hat{I}_{K}^{m a}$ is an equilibrium. The change to malls therefore increases equilibrium $\hat{I}_{K}^{m}$.

The second conversion that we consider, a change from streets to supermarkets, is more complex because the direct effect is now influenced by retailer internalization. Suppose initially all centers are in street equilibrium, setting $\hat{I}_{K}^{s t}$ so $\delta^{s t}\left(\hat{I}_{K}^{s t}, 1 / K\right)=0$. As before, developer internalization takes $y^{m}$ from 1 to $I_{k}$, extending the developer's market share effect to all products. But this is combined with retailer internalization, which reduces $\hat{\pi}_{K}^{m}$, diminishing the direct effect $\hat{\pi}_{K}^{m}-f^{\prime}$ (and ambiguously impacting the market share effect). Thus, the positive impact of developer internalization on the market share effect is now coupled with the negative impact of retail internalization on the direct effect, so the change in $\delta^{s u}\left(\hat{I}_{K}^{s t}, 1 / K\right)$ is ambiguous, suggesting the possibility that $\hat{I}_{K}^{m}$ may rise or fall with a conversion from streets to supermarkets.

Example 2 (Cases $\mathrm{A}$ and $\mathrm{B}$ ) shows that the number of product lines may indeed rise or fall $\left(\hat{I}_{K}^{s u} \lessgtr \hat{I}_{K}^{s t}\right)$. The difference between the two cases is in the shape of the demand function $q()$. We now sketch the intuition for the results informally. (The assumptions of the model are detailed below, and the results are fully worked out in Smith, 2004.) For simplicity quality $n_{k}$ is exogenously fixed so the increase in $u_{k}$ in supermarket mode is achieved through a fall in $p_{k}$. In Case A the demand function $q\left(\right.$ ) is concave (at a kink) such that price elasticity falls as $p_{k}$ falls, which reduces the supermarket's incentive to cut $p_{k}$, limits the fall in $\hat{\pi}_{K}^{m}$, and moderates the change in the direct effect. The developer's market share effect dominates the change in the direct effect, and supermarkets increase $\hat{I}_{K}^{m}$. In the limit, as the effect on price goes to zero, the conversion is identical to the change from streets to malls, where $\hat{I}_{K}^{m}$ increases. In Case B the argument is reversed. Now demand $q()$ is convex, so price elasticity increases as $p_{k}$ falls, magnifying the supermarket's incentive to cut $p_{k}$ and aggravating the fall in $\hat{\pi}_{K}^{m}$. If this effect is large enough, as for the parameter values in Case $B$, the fall in the direct effect dominates and the change from streets to supermarkets reduces $\hat{I}_{K}^{m}$.

Finally, consider a change from malls to supermarkets. Here developers internalize in both modes, so that the only change is the introduction of retailer internalization. Suppose all centers are initially malls, setting $\delta^{m a}\left(\hat{I}_{K}^{m a}, 1 / K\right)=0$. We know from the previous section that the introduction of retailer internalization increases $\hat{u}_{K}^{m}$ and reduces $\hat{\pi}_{K}^{m}$. This has two impacts on the developer's condition (12): a fall in the direct effect $\hat{\pi}_{K}^{m}-f^{\prime}$ and a rise, through $\hat{u}_{K}^{m}$, in the market share effect. (A third effect is that $\partial \hat{\pi}_{k}^{m} / \partial s_{k}$ in the market share effect rises or falls.) 
The overall effect on $\delta^{m a}\left(\hat{I}_{K}^{s u}, 1 / K\right)$ is ambiguous, suggesting $\hat{I}_{K}^{m}$ may rise or fall. The direction depends on the relative size of the changes in $\hat{u}_{K}^{m}$ and $\hat{\pi}_{K}^{m}$, determined again by the shape of $q($ ).

Example 2 (Cases B and C) confirms that the change from malls to supermarkets may increase or reduce the number of product lines $\left(\hat{I}_{K}^{s u} \lessgtr \hat{I}_{K}^{m a}\right)$. Again, the difference between the two cases hinges on the shape of the retailer's demand function $q($ ). In Case B, as noted above, the demand function is convex, so that the supermarket mode takes $p_{k}$ to a more elastic portion of demand, aggravating the fall in $\hat{\pi}_{K}^{m}$. The fall in the direct effect dominates other effects and the developer sets a lower number of product lines in supermarket mode than in mall mode $\left(\hat{I}_{K}^{s u}<\hat{I}_{K}^{m a}\right)$. In Case $C$ demand $q()$ is everywhere zero-elastic, except for a discontinuity at a given price below which there is a step increase in demand. In the mall mode, $p_{k}$ is above this point and is close to the consumer's reservation price, so that $\hat{u}_{K}^{m a}$ is very small. The supermarket mode takes $p_{k}$ below the step increase in demand, resulting in a large increase in $\hat{u}_{K}^{m}$, and a large increase in the market share effect, dominating the fall in the direct effect so that $\hat{I}_{K}^{m}$ increases. Proposition 5 summarizes.

PROPOSITION 5: In symmetric equilibrium with given $K$ (i) a change from streets to malls increases $\hat{I}_{K}^{m}$ (i.e., $\hat{I}_{K}^{m a} \geq \hat{I}_{K}^{s t}$ ); (ii) a change from streets to supermarkets may increase or reduce $\hat{I}_{K}^{m}$ (i.e., $\hat{I}_{K}^{s u} \lessgtr \hat{I}_{K}^{s t}$ ); (iii) a change from malls to supermarkets may increase or reduce $\hat{I}_{K}^{m}\left(\right.$ i.e., $\left.\hat{I}_{K}^{s u} \lessgtr \hat{I}_{K}^{m a}\right)$.

Proof. (i) Let $\hat{I}_{K}^{m}$ denote the unique symmetric equilibrium $I_{k}$ solving $\delta^{m}\left(I_{k}, 1 / K\right)=0$ for any $K$ and any $m$. The effect of $m$ on $\delta^{m}()$ is exclusively through $y^{m}$, which increases from 1 to $\hat{I}_{K}^{m a}$ when $m$ changes from $s t$ to $m a$. By strict positive monotonicity of $\pi_{k}()$ in $s_{k}$, and $s_{k}()$ in $v_{k}$, the second term in (12) is positive, so $\delta_{k}^{m}()$ is increasing in $y^{m}$. This gives $\delta_{k}^{m a}\left(\hat{I}_{K}^{s t}, 1 / K\right)>0$ and, by the second line in $(7), \delta_{k}^{m a}\left(\hat{I}_{K}^{m a}, 1 / K\right)=0$ is satisfied only for $\hat{I}_{K}^{m a}>\hat{I}_{K}^{s t}$. (ii) See Example 2 Cases A and B. (iii) See Example 2 Cases B and C.

EXAMPLe 2 [Symmetric Salop Circular-City]: Let consumers be uniformly distributed in a circle city of unit circumference with $K$ evenly spaced shopping centers. Let transport costs $t$ be linear, giving $\partial s_{k} / \partial v_{k}=$ $1 / t$. Let $n_{k}$ be fixed exogenously, and let retailer costs be $\left\{c_{k}=0, g_{k}=0\right.$, $\left.f_{k}=0.5 I_{k}^{2}\right\}$. Let each consumer's demand $q_{k}$ be

$q\left(p_{k}\right)=\left\{\begin{array}{cc}1 & \text { for } 1<p_{k} \leq a \\ b p_{k}^{-\theta} & \text { for } 0<p_{k} \leq 1 .\end{array}\right.$

Above $a$ elasticity exceeds 1 , so $\hat{p}_{K}^{m} \leq a$. The area below the inverse demand, up to $q_{k}=1$, is $A>a$. Case A: Parameters are $\{\theta=0, b=$ $\left.1, s_{k} t>a>t / A\right\}$ giving $\hat{I}_{K}^{s t}<\hat{I}_{K}^{s u}$. Case B: Parameters are $\{\theta>0, b=1$, 
$\left.a>s_{k} t>1 /(1-\theta), t / A<1\right\}$ giving $\hat{I}_{K}^{m a}>\hat{I}_{K}^{s t}>\hat{I}_{K}^{s u}$. Case C: Parameters are $\left\{\theta=0, b>1, a>s_{k} t>b, t / A<1\right\}$ giving $\hat{I}_{K}^{s u}>\hat{I}_{K}^{m a}$. (For a derivation of these results see Smith, 2004.)

\section{Number OF CENTERS}

For any number of centers $K$, the symmetric equilibrium profit $\Pi_{K}^{m}$ at any center, with $\hat{I}_{K}^{m}$ products each giving utility $\hat{u}_{K}^{m}$, is given by retail profit minus developer fixed costs $f$, i.e.,

$\Pi_{K}^{m}=\hat{I}_{K}^{m} \pi\left(\hat{u}_{K}^{m}, 1 / K\right)-f\left(\hat{I}_{K}^{m}\right)$.

Centers enter until $\Pi_{K}^{m}$ declines to zero, so the effect of mode on freeentry center numbers $\hat{K}^{m}$ is through $\Pi_{K}^{m}$, treating $K$ as a continuous variable. In this section we compare the equilibrium number of centers in each mode.

Conversion of streets to malls implies no change in retailer conduct, which means that utility $\hat{u}_{K}^{m}$ and profit $\hat{\pi}_{K}^{m}$ on each product line are unchanged. However, developers expand the number of product lines $\hat{I}_{K}^{m}$, being prepared to give up more profit (than before) on current customers in return for any increase in $s_{k}$ (see Proposition 5). In symmetric equilibrium, when all centers increase $\hat{I}_{K}^{m}$, the market share effects cancel, and profit $\Pi_{K}^{m}$ falls by the extent of the profit forgone. This reduces the number of centers $\hat{K}^{m}$.

Conversion of streets to supermarkets implies both retailer and developer internalization. Both are now prepared to sacrifice more profit on current customers in return for any given increase in $s_{k}$. Therefore, in symmetric equilibrium, when market share effects cancel out, $\Pi_{K}^{m}$ is lower, reducing the free-entry number of centers $\hat{K}^{m}$.

In a conversion from malls to supermarkets, developer internalization is maintained, and retail internalization is introduced, extending the retailer's market share effect to all products at a center. For any $\hat{I}_{K}^{m}$ this reduces $\hat{\pi}_{K}^{m}$ as retail conduct becomes more competitive. If $\hat{I}_{K}^{m}$ is unchanged there is a reduction in developer profit $\Pi_{K}^{m}$. If $\hat{I}_{K}^{m}$ increases, $\Pi_{K}^{m}$ also falls (in symmetric equilibrium), as marginal product lines have a negative direct effect. However, if retailer internalization reduces $\left(\hat{p}_{K}^{m}-c_{k}\right) q_{k}$, the developer's market share effect falls, reducing $\hat{I}_{K}^{m}$, because the developer is now prepared to sacrifice less in return for any increase in $s_{k}$. Consequently, there is an ambiguous effect on $\Pi_{K}^{m}$ in symmetric equilibrium, and ambiguous consequences for $\hat{K}^{m}$. Proposition 6 summarizes results (omitting the inconclusive comparison between malls and supermarkets).

Proposition 6: (A) In symmetric equilibrium with given $\mathrm{K}$ : (i) a change from streets to malls reduces center profit $\left(\Pi_{K}^{m a}<\Pi_{K}^{s t}\right)$; (ii) a change from 
streets to supermarkets reduces center profit $\left(\Pi_{K}^{s u}<\Pi_{K}^{s t}\right)$. (B) In symmetric free-entry equilibrium: (i) a change from streets to malls reduces center numbers $\left(\hat{K}^{m a}<\hat{K}^{s t}\right)$; (ii) a change from streets to supermarkets reduces center numbers $\left(\hat{K}^{s u}<\hat{K}^{s t}\right)$.

Proof. (A) (i) With $K$ centers, symmetric equilibrium center profits for mall and street mode are $\Pi_{K}^{m a}=\hat{I}_{K}^{m a} \pi\left(\hat{u}_{K}^{m a}, 1 / K\right)-f\left(\hat{I}_{K}^{m a}\right)$ and $\Pi_{K}^{s t}=$ $\hat{I}_{K}^{s t} \pi\left(\hat{u}_{K}^{s t}, 1 / K\right)-f\left(\hat{I}_{K}^{s t}\right)$. By Proposition $5, \hat{I}_{K}^{m a} \geq \hat{I}_{K}^{s t}$. At any $K$ malls and streets have identical $\rho^{m}\left(\right.$ ) (see (8)), so $\hat{u}_{K}^{m a}=\hat{u}_{K}^{s t}$ is independent of $\hat{I}_{K}^{m}$. By condition (12), $\hat{\pi}_{K}^{s t}-f^{\prime}\left(\hat{I}_{K}^{s t}\right)<0$ so that since $f^{\prime \prime}()>0$ and $\hat{u}_{K}^{m a}=\hat{u}_{K}^{s t}$ it follows that $\left(\hat{I}_{K}^{m a}-\hat{I}_{K}^{s t}\right) \hat{\pi}_{K}^{m a}-\left[f\left(\hat{I}_{K}^{m a}\right)-f\left(\hat{I}_{K}^{s t}\right)\right]<0$. Thus $\Pi_{K}^{m a}<\Pi_{K}^{s t}$. (ii) For any $K$ let conversion from streets to supermarkets occur in a series of increments to $x^{m}$ at all centers, from $x^{m}=1$ to $x^{m}=\hat{I}_{K}^{s u}$. At each $x^{m}$ we maintain $y^{m}=x^{m}$ and retailers and developers set $\hat{u}_{K}^{m}$ and $\hat{I}_{K}^{m}$ to satisfy the first-order condition for $\hat{v}_{K}^{m}=\hat{u}_{K}^{m} \hat{I}_{K}^{m}$

$\theta^{m}\left(\hat{u}_{K}^{m}, 1 / K\right)=\frac{\partial \Pi_{k}^{m}}{\partial v_{k}}+x^{m} \frac{\partial \pi_{k}}{\partial s_{k}} \frac{\partial s_{k}}{\partial v_{k}}=0$.

Because $\frac{\partial \pi_{k}}{\partial s_{k}} \frac{\partial s_{k}}{\partial v_{k}}>0$ we have $\frac{\partial \Pi_{k}^{m}}{\partial v_{k}}<0$, and $\theta^{m}()$ increasing in $x^{m}$, for all $x^{m}$ between 1 and $\hat{I}_{K}^{s u}$, so that $\frac{\partial v_{K}^{m}}{\partial x_{k}}>0$, implying that $\frac{\partial \Pi_{k}^{m}}{\partial v_{k}} \frac{\partial \hat{v}_{k}^{m}}{\partial x_{k}}<0$ for all $x^{m}$ between 1 and $\hat{I}_{K}^{s u} \cdot \frac{\partial \Pi_{k}^{m}}{\partial v_{k}} \frac{\partial \hat{v}_{k}^{m}}{\partial x_{k}}$ is the marginal effect of $x^{m}$ on $\Pi_{k}^{m}$ holding $s_{k}=1 / K$, so $\Pi_{K}^{s u}<\Pi_{K}^{s t}$.

(B) Let $\hat{K}^{m}$ denote free-entry $K$ in mode $m$ satisfying $\Pi_{K}^{m}=0$. Note from (14) that $\Pi_{K}^{m}$ is increasing in $s_{k}$ and therefore decreasing in $K$. (i) By A(i) we have $\Pi_{K}^{m a}<\Pi_{K}^{s t}$ for each $K$. Because $\Pi_{K}^{m a}$ and $\Pi_{K}^{s t}$ are decreasing in $K, \hat{K}^{m a}<\hat{K}^{s t}$. (ii) By A(ii) we have $\Pi_{K}^{s u}<\Pi_{K}^{s t}$ for each $K$. Since $\Pi_{K}^{s u}$ and $\Pi_{K}^{s t}$ are decreasing in $K, \hat{K}^{s u}<\hat{K}^{s t}$.

\section{Developer Choice of Mode}

Up to now we have taken as given that centers are a common mode and we continue with this assumption in the next section. In this section we deviate from this to discuss informally the best choice of $m_{k}$ for the entering developer, or consortium of developers, at center $k$, given the common mode of other entering centers $m_{-k}$. For simplicity we fix $I$ and $K$ so malls are identical to streets and we may therefore suppress any mention of malls.

As we have seen, center $k^{\prime}$ s best reply in $\hat{u}_{k}^{m}$ shifts upward in supermarket mode. We also know that an increase in $\hat{u}_{k}^{m}$ reduces $\hat{\pi}_{k}^{m}$ for fixed $s_{k}$, but increases $\hat{\pi}_{k}^{m}$ if $s_{k}$ grows sufficiently. The supermarket mode is therefore $k^{\prime}$ s best choice (for any $m_{-k}$ ) provided $s_{k}$ grows sufficiently. 
This is determined by the system of best reply functions for $\hat{\mathbf{u}}^{m}$. Typically these are upward sloping. The flatter they are, the smaller the change in $\hat{\mathbf{u}}_{-k}$, and the larger the increase in $s_{k}$. In the limit, with flat best reply functions so $\hat{\mathbf{u}}_{-k}$ is fixed, the supermarket mode is $k^{\prime}$ s best choice, for the familiar reason that externalities are internalized. At the other extreme, however, with very steep best reply functions in $\hat{\mathbf{u}}^{m}$, the increase in $s_{k}$ is negligible, and street mode is $k^{\prime}$ s best choice. Example 3 shows that $k^{\prime} \mathrm{s}$ best choice depends on best reply functions. Thus, if all centers were free to change mode, there are some parameter values for which each mode is not a symmetric (perfect) equilibrium.

EXAMPLe 3 [Logit Oligopoly]: Let $K=5$ and $I_{k}=2 . n_{k}$ is fixed such that $\left\{c_{k}=0, g_{k}=0\right\}$. Demand is zero-elastic at $q_{k}=1$ and $u_{k}$ from each good is $u_{k}=\alpha-b p_{k}$. Transport cost is $\varepsilon_{k}=t \zeta_{k}$ where $\zeta_{k}$ is a Type- 1 EV deviate and $t=5$ is a scaling term, giving standard logit oligopoly results,

$\hat{p}_{K}^{m}=t / b x^{m}\left(1-s_{k}\right) \quad s_{k}=e^{v_{k}} / \sum_{l=1}^{K} e^{v_{l}}$

where $\hat{p}_{K}^{m}$ uses $\partial s_{k} / \partial v_{k}=s_{k}\left(1-s_{k}\right) / t$ and there is no "outside good." Table I shows $s_{k}, \hat{p}_{K}^{m}$, and $\Pi_{K}^{m}$ for center $k$ in four cases: $(s t) s t$ where all centers are streets, $(s t) s u$ where other centers are streets and $k$ is a supermarket, $(s u) s t$ where other centers are supermarkets and $k$ is a street, and $(s u) s u$ where all centers are supermarkets. The rows give results for three values of parameter $b$. Center $k$ 's best mode choice is in square brackets []. First, with $b=0.5$ consumers are price insensitive and best reply functions are relatively flat. Here $k$ chooses the supermarket mode whether other centers are streets or supermarkets. Second is an intermediate case $b=0.65$, where $k$ matches the mode of the other centers. Third, with $b=0.8$, consumers are price sensitive, best reply

TABLE I.

EXAMPle 3: Center $k$ Choice of Mode*

\begin{tabular}{|c|c|c|c|c|c|c|c|c|c|c|c|c|}
\hline \multirow{3}{*}{$\begin{array}{l}\mathrm{m}_{-k} \\
\mathrm{~m}_{k}\end{array}$} & \multicolumn{4}{|c|}{ Market Share } & \multicolumn{4}{|c|}{ Prices } & \multicolumn{4}{|c|}{ Retailer Profit at Center } \\
\hline & \multicolumn{2}{|c|}{ (st) } & \multicolumn{2}{|c|}{ (su) } & \multicolumn{2}{|c|}{ (st) } & \multicolumn{2}{|c|}{ (su) } & \multicolumn{2}{|c|}{ (st) } & \multicolumn{2}{|c|}{ (su) } \\
\hline & st & $\mathrm{su}$ & st & $\mathrm{su}$ & st & su & st & $\mathrm{su}$ & st & su & st & su \\
\hline $\mathrm{b}=0.50$ & 0.20 & 0.36 & 0.09 & 0.20 & 12.5 & 7.83 & 11.0 & 6.25 & 5.00 & {$[5.65]$} & 2.02 & [2.50] \\
\hline$b=0.65$ & 0.20 & 0.11 & 0.33 & 0.20 & 9.61 & 5.77 & 8.61 & 4.81 & [3.85] & 3.84 & 1.85 & [1.92] \\
\hline $\mathrm{b}=0.80$ & 0.20 & 0.12 & 0.31 & 0.20 & 7.81 & 4.55 & 7.09 & 3.91 & [3.13] & 2.85 & [1.69] & 1.56 \\
\hline
\end{tabular}

* Round brackets indicate mode of other centers.

Square brackets indicate $k^{\prime}$ s optimal mode. 
functions are steep, and $k$ chooses the street mode, whether other centers are streets or supermarkets. The results imply that, if all centers were free to change mode, (st)st is not a (symmetric) equilibrium at $b=0.5$ and $(s u(s u))$ is not a (symmetric) equilibrium at $b=0.8$.

\section{SOCIAL EFFICIENCY}

Social welfare $W$ is consumer utility minus consumer transportation costs $T$ plus the profit of the $K$ centers. Recalling that we have scaled the number of consumers to 1 , welfare may be written as follows, in a symmetric equilibrium:

$$
W=\hat{v}_{K}^{m}-T(K)+K \Pi_{K}^{m}
$$

where transportation costs $T()$ are declining in $K$. In this section we first examine whether retail internalization increases $W$, for fixed values of the product range $I_{k}$ and the number of centers $K$. Then, we allow developers to set $\hat{I}_{K}^{m}$ and compare $W$ for alternative modes $m$, given a fixed number of centers $K$. Finally, we compare $W$ for alternative modes $m$, with $\hat{I}_{K}^{m}$ set by developers and $K$ determined by free entry.

\subsection{RETAiLER INTERNALIZATION}

Assuming fixed values of $I_{k}$ and $K$ we compare streets and supermarkets. (Since $I_{k}$ is fixed in this subsection, malls are identical to streets, and the results for streets also apply to malls.) When setting $\hat{u}_{K}^{m}$ on any product to maximize private profit, through choice of $p_{k}$ and $n_{k}$, a retailer imposes effects on consumers and rival retailers. If a small increase in utility, from equilibrium level $\hat{u}_{K}^{m}$, increases consumer surplus more than it reduces rival retailer surplus, then $\hat{u}_{K}^{m}$ is too low relative to the social optimum (the Spence, 1976 condition).

With one-stop shopping, the effect on rival retailers, known as the business stealing effect, differs from the standard oligopoly case because consumers transfer all product demands to center $k$. This has two consequences. First, a negative externality is experienced on all products at rival centers. Second, in the street mode, there is a positive external effect (a "reverse business stealing" effect) on center $k$ 's other retailers, reducing net business stealing-i.e., business stealing net of reverse business stealing. By symmetry, a retailer's net business stealing is identical to its market share effect (in (8)) for either mode: for a supermarket the market share and business stealing effects are the profits transferred to center $k$ on all products, for a street they are the 
profit transferred on the retailer's own product (business stealing effects on other products being offset by reverse business stealing).

We differentiate (16) to get the marginal effect of $u_{k}$ (for any given product at center $k$ ),

$\frac{\partial W}{\partial u_{k}}=s_{k}+\left(\frac{\partial \pi_{k}}{\partial u_{k}}\right)_{s_{k}}$

where the first term is the consumer surplus effect, given $s_{k}$, and the second is the direct effect on profit, given $s_{k}{ }^{12}$ In equilibrium, the retailer increases $u_{k}$ until the direct effect exactly offsets the market share effect, which is (identically) net business stolen. Thus (17) is the Spence condition, as shown by substituting from (8) into (17) at $\rho_{k}^{m}=0$ to obtain

$$
\frac{\partial W}{\partial u_{k}}=\left[s_{k}-x^{m} \frac{\partial \pi_{k}}{\partial s_{k}} \frac{\partial s_{k}}{\partial u_{k}}\right]
$$

where the second term is net business stolen.

Utility $\hat{u}_{K}^{m}$ is suboptimal; the consumer surplus effect outweighs the direct effect. This can be established for either $m$. The direct effect is the retailer's cost in profit forgone of increasing $u_{k}$; at most this is the cost of increasing $u_{k}$ by cutting $p_{k}$, since firms set $u_{k}$ by the most profit-maximizing means available. As the increase in consumer surplus from cutting $p_{k}$ always exceeds profit foregone (or equals it with vertical demand), $\hat{u}_{K}^{m}$ is less than the socially optimal level (or equals it with vertical demand).

Because streets and supermarkets both underprovide $\hat{u}_{K}^{m}$ (except with vertical demand) and conversion from streets to supermarkets increases $\hat{u}_{K}^{m}$, the conversion increases $W$ (or leaves it unchanged, with vertical demands). Proposition 7 summarizes.

Proposition 7: (A) In symmetric equilibrium with given values of $m, I_{k}$, and $K$, utility $\hat{u}_{K}^{m}$ is less than the social optimum (or with vertical demand equals the social optimum). (B) Assuming given values of $I_{k}$ and $K$, a change from streets (or malls) to supermarkets increases equilibrium W (or with vertical demand has no effect on $W$ ).

Proof. Because $I$ is fixed, the results for streets also apply to malls. (A) For $s_{k}=1 / K$ we show $\partial W / \partial u_{k} \geq 0$ at $\hat{u}_{K}^{m}$ satisfying

$\rho_{k}^{m}=\left(\frac{\partial \pi_{k}}{\partial u_{k}}\right)_{s_{k}}+s_{k} z^{m}=0 \quad$ for any $m$

12. The effect of change in $s_{k}$ is zero by symmetry because all centers have identical gross profits per customer, and marginal consumers are indifferent between centers. 
where $z^{m}=\frac{x^{m}}{s_{k}} \frac{\partial s_{k}}{\partial v_{k}}\left\{q_{k}\left(\hat{p}_{k}^{m}-c_{k}\right)\right\}$. By (16),

$\frac{\partial W}{\partial u_{k}}=s_{k}+\left(\frac{\partial \pi_{k}}{\partial u_{k}}\right)_{s_{k}}$

at symmetric $\hat{u}_{K}^{m}$, where the effect on $W$ through a change in $s_{k}$ is zero (by symmetry of gross profits across centers). Substituting from (19) at $\hat{u}_{K}^{m}$,

$\frac{\partial W}{\partial u_{k}}=\rho_{k}^{m}+s_{k}\left[1-z^{m}\right]$

where profit maximization implies $\rho_{k}^{m}=0$ and $0<z^{m} \leq 1$ (Lemma 1, Appendix) giving $\partial W / \partial u_{k} \geq 0$. If and only if $q()$ is zero price-elastic then $z^{m}=1$ (Lemma 2, Appendix) and this is the only case where $\partial W / \partial u_{k}=0$. (B) We show $d W / d u_{k} \geq 0$ at $s_{k}=1 / K$ for each $u_{k}$ (identical across lines and centers) between $\hat{u}_{K}^{s t}$ and $\hat{u}_{K}^{s u}$. By continuity of (19) in $x^{m}$ and $u_{k}$, and monotonicity in $x^{m}$, there is always a notional level of $x^{m}$ between 1 and $\hat{I}_{K}^{s u}$ at which $\rho_{k}^{m}=0$ for each $u_{k}$ (identical across lines and centers) between $\hat{u}_{K}^{s t}$ and $\hat{u}_{K}^{s u}$. By profit maximization at this $x^{m}, \rho_{k}^{m}=0$ and $0<$ $z^{m} \leq 1$ (Lemma 1), and (21) gives $\partial W / \partial u_{k} \geq 0$. If and only if $q()$ is zero price-elastic $z^{m}=1$ (Lemma 2 ) and this the only case where $\partial W / \partial u_{k}=0$.

\subsection{DEVELOPER INTERNALIZATION}

For fixed $K$ we now compare modes, allowing $\hat{I}_{K}^{m}$ to be chosen by developers (and $\hat{u}_{K}^{m}$ by retailers). The developer imposes effects on consumers and other developers, underproviding $\hat{I}_{K}^{m}$ if benefits to consumers outweigh net negative effects on other developers (see Spence, 1976). Because $I_{k}$ is endogenous, streets and malls are no longer identical, and we compare all three modes.

With one-stop shopping, a marginal product line at center $k$ steals business on all products at rival centers, and, in the street mode, gives business to other developers at center $k$ ("reverse business stealing"). The business stealing effect of a line, net of reverse business stealing, equals its market share effect: in a supermarket this is the profit transferred to center $k$ on all I products; in a street it is the profit transferred on the product at hand (other product's business stealing effects being exactly offset by reverse business stealing).

Imagine $K$ centers of mode $m$ in a symmetric equilibrium with product range $\hat{I}_{K}^{m}$ and utility $\hat{u}_{K}^{m}$ on each line. The marginal line increases 
an individual consumer's utility by $\hat{u}_{K}^{m}$. Consider the marginal line at center $k$. Differentiating (16) we obtain its effect on social welfare $W$,

$\frac{\partial W}{\partial I_{k}}=s_{k} \hat{u}_{K}^{m}+\left[\pi\left(\hat{u}_{K}^{m}, s_{k}\right)-f^{\prime}\left(\hat{I}_{K}^{m}\right)\right]$

where the first term is the consumer surplus effect, given $s_{k}$, and the final two terms are the direct effect on center $k$ 's profits, given $s_{k} \cdot{ }^{13}$ Because a profit-maximizing developer sets direct effect equal to market share effect, and the market share effect is identical to the net business stealing effect, it follows that (22) is the familiar Spence criterion comparing consumer surplus and business stealing effects, as may be seen by substituting into (22) from (12) at $\delta_{k}^{m}=0$ to give

$$
\frac{\partial W}{\partial I_{k}}=s_{k} \hat{u}_{K}^{m}-y^{m} \frac{\partial \pi_{k}}{\partial s_{k}} \frac{\partial s_{k}}{\partial v_{k}} \hat{u}_{K}^{m}
$$

the second term being net business stolen.

We now assess whether the $\hat{I}_{K}^{m}$ th line is socially excessive by comparing its direct and consumer surplus effects for each mode.

First consider the two modes where developers and retailers both internalize to the same extent, i.e., streets (where neither internalize and $x^{s t}=y^{s t}=1$ ) and supermarkets (where both internalize and $x^{s u}=$ $y^{s u}=\hat{I}_{K}^{s u}$ ) but not malls (where developers internalize but retailers do not $\hat{I}_{K}^{m a}=y^{m a}>x^{m a}=1$ ). In each of these modes (supermarkets and streets), since the developer's profit is appropriated entirely from the retailer, developer and retailer have identical market share effects from any increase in $u_{k}$, and are therefore willing to sacrifice the same direct effect to achieve it. The retailer's direct effect is at most the cost of offering the utility by cutting $p_{k}$, which never exceeds the consumer surplus generated. Because the developer incurs the same direct effect as the retailer, this too never exceeds the consumer surplus generated, so $\hat{I}_{K}^{m}$ is less than or equal to the social optimum. (The case of equality applies only with vertical demands when consumer surplus equals the profit forgone.) To see that (23) is nonpositive, note that with $y^{m}=x^{m}$ it has the same sign as (18), which we established to be nonpositive in the previous subsection.

Now consider malls. Here, developers internalize but retailers do not $\left(\hat{I}_{K}^{m a}=y^{m a}>x^{m a}=1\right)$, and the developer's profit exceeds that of any one of the retailers, violating the argument just used for streets and supermarkets. For a unit increase in $u_{k}$ at center $k$, the developer's direct effect is now $\hat{I}_{K}^{m a}$ times any retailer's direct effect. We know that the latter is bounded above by the gain to consumers (equaling it for

13. As in the previous subsection, the effect of change in $s_{k}$ is zero by symmetry since all centers have identical gross profits per customer, and marginal consumers are indifferent between centers. 
vertical demands). However, because $y^{m a}>x^{m a}$, the developer's direct effect is not bounded by the consumer gain, so that $\hat{I}_{K}^{m a}$ may now be socially excessive. In effect, the developer's business stealing effect has been expanded, for any given consumer surplus effect, and may now dominate. To see this, note that when $y^{m a}>x^{m a}(23)$ may be less than (18), and may be negative. For instance, with vertical demands the retailer's direct effect equals the consumer surplus effect, the developer's direct effect is $\hat{I}^{m a}$ times this, so that $\hat{I}_{K}^{m a}$ is excessive.

We now ask whether internalization is welfare-improving, with endogenous $I_{k}$.

Consider a change from streets to supermarkets, taking place in a series of increments to $x^{m}$ and $y^{m}$, from 1 to $\hat{I}_{K}^{s u}$, maintaining $y^{m}=x^{m}$ throughout. Each increase augments the market share effect of both retailers and developers, increasing $\hat{v}_{K}^{m}$. Because developers and retailers have the same market share effect (as each other) from an increase in $v_{k}$, they share the same direct effect, which is always less than (or equal to, with vertical demand) the consumer surplus gain. Thus the consumer surplus effect dominates, and there is an increase in $W$ (or no change, with vertical demand).

The argument does not hold for conversion from streets to malls. Here $y^{m}$ increases from 1 to $\hat{I}_{K}^{m}$ while $x^{m}$ remains at 1 . This extends the developer's business stealing effect from any increase in $v_{k}$, but this is not now tied to the retailer's direct effect, so there is no guarantee it is bounded above by the consumer surplus effect. (With vertical demand, the consumer surplus effect of any utility increase equals the retailer's direct effect, so the developer's direct effect is $\hat{I}_{K}^{m}$ times this, and the additional lines offered by the mall reduce $W$ ). Proposition 8 summarizes.

Proposition 8: (A) For any fixed $K$, symmetric equilibrium $\hat{I}_{K}^{m}$ (i) may exceed the social optimum in malls (and does if demands are vertical), and (ii) is less than the social optimum in streets and supermarkets (or equals it if demands are vertical). (B) For given $K$, (i) a change from streets to malls may reduce welfare (and does if demands are vertical), and (ii) a change from streets to supermarkets increases welfare (or is unchanged if demands are vertical).

Proof. (A) For $s_{k}=1 / K$ we find the sign of $\partial W / \partial I_{k}$ at $\hat{I}_{K}^{m}$ satisfying

$\delta_{k}^{m}=\pi\left(\hat{u}_{K}^{m}, s_{k}\right)-f^{\prime}\left(\hat{I}_{K}^{m}\right)+\frac{y^{m}}{x^{m}} s_{k} z^{m} \hat{u}_{K}^{m}=0 \quad$ for any $m$

where $z^{m}=\frac{1}{s_{k}} \frac{\partial s_{k}}{\partial v_{k}} x^{m}\left\{q_{k}\left(\hat{p}_{K}^{m}-c_{k}\right)\right\}$. By (16),

$\frac{\partial W}{\partial I_{k}}=s_{k} \hat{u}_{K}^{m}+\pi\left(\hat{u}_{K}^{m}, s_{k}\right)-f^{\prime}\left(\hat{I}_{K}^{m}\right)$ 
at symmetric $\hat{I}_{K}^{m}$, where effects through $s_{k}$ are zero by symmetry of gross profits across centers. Substituting from (24),

$$
\frac{\partial W}{\partial I_{k}}=\delta_{k}^{m}+\left[1-\frac{y^{m}}{x^{m}} z^{m}\right] s_{k} \hat{u}_{K}^{m}
$$

where, by profit maximization, $\delta_{k}^{m}=0$ and $0<z^{m} \leq 1$ (Lemma 1, Appendix). Part (i): in malls $y^{m a} / x^{m a}=\hat{I}_{K}^{m a}$ so that for $z^{m a}>1 / \hat{I}_{K}^{m a}$ we get $\partial W / \partial I_{k}<0$. If and only if $q()$ is zero price-elastic, $z^{m}=1$ (Lemma 2, Appendix), giving a case where $\partial W / \partial I_{k}<0$. Part (ii): in streets and supermarkets $x^{m}=y^{m}$, so $\partial W / \partial I_{k} \geq 0$. If and only if $q()$ is zero priceelastic, $z^{m}=1$ (Lemma 2 ), and the only case where $\partial W / \partial I_{k}=0$.

(B) Part (i): to show that $W$ may fall in the mall mode, we give the conditions for $d W / d I_{k}<0$ at $s_{k}=1 / K$ for each $I_{k}$ (identical across centers) between $\hat{I}_{K}^{s t}$ and $\hat{I}_{K}^{m a}$. By continuity of (24) in $y^{m}$ and $I_{k}$, and monotonicity in $y^{m}$, there is always some notional level of $y^{m}$ between 1 and $\hat{I}_{K}^{m a}$ that satisfies $\delta_{k}^{m}=0$, for each $I_{k}$ (identical across centers) between $\hat{I}_{K}^{s t}$ and $\hat{I}_{K}^{m a}$. By profit maximization at this $y^{m}, \delta_{k}^{m}=0$ and $0<z^{m} \leq 1$ (Lemma 1 ) so (26) gives $d W / d I_{k}<0$ when $z^{m} y^{m}>1$ (recalling that $x^{m}=1$ throughout in the mall mode). If and only if $q()$ is zero price-elastic, $z^{m}=1$ (Lemma 2 ) providing an example where $\partial W / \partial I_{k}<0$, because $y^{m}>1$ (and $x^{m}=1$ ). Part (ii): to show $W$ increases in the supermarket mode, let $y^{m}$ increase from $y^{s t}=1$ to $y^{s u}=\hat{I}_{K}^{s u}$ with $x^{m}=y^{m}$, such that for each $y^{m}, \hat{u}_{K}^{m}$ and $\hat{I}_{K}^{m}$ satisfy the condition for $\hat{v}_{K}^{m}=\hat{u}_{K}^{m} \hat{I}_{K^{\prime}}^{m}$

$\theta_{k}^{m}=\theta^{m}\left(\hat{v}_{K}^{m}, 1 / K\right)=\left(\frac{\partial \Pi_{k}^{m}}{\partial v_{k}}\right)_{s_{k}}+y^{m} \frac{\partial \pi_{k}}{\partial s_{k}} \frac{\partial s_{k}}{\partial v_{k}}=0$.

Because $\frac{\partial \pi_{k}}{\partial s_{k}} \frac{\partial s_{k}}{\partial v_{k}}>0, \theta^{m}()$, and therefore $\hat{v}_{K}^{m}$, are increasing in $y^{m}$. By (16), $\frac{\partial W}{\partial v_{k}}=s_{k}+\left(\frac{\partial \Pi_{k}^{m}}{\partial v_{k}}\right)_{s_{k}}=\theta_{k}^{m}+\left[1-z^{m}\right] s_{k}$

by substitution from (27) where $z^{m}=\frac{1}{s_{k}} \frac{\partial s_{k}}{\partial v_{k}} x^{m} q_{k}\left(\hat{p}_{k}^{m}-c_{k}\right)$ and $x^{m}=y^{m}$. At each symmetric $\hat{v}_{K}^{m}$ between $\hat{v}_{K}^{s t}$ and $\hat{v}_{K}^{s u}$ profit maximization implies $\theta_{k}^{m}=0$ and $0<z^{m} \leq 1$ (Lemma 1 ) so $\frac{\partial W}{\partial v_{k}} \geq 0$. If and only if $q()$ is zero price-elastic then $z^{m}=1$ (Lemma 2) and $\frac{\partial W}{\partial v_{k}}=0$.

\subsection{FREE ENTRY}

In this subsection we discuss the welfare effects of mode in free-entry equilibrium. We compare streets with supermarkets and malls. (We omit the inconclusive comparison of malls and supermarkets.) We now let $y$ denote the level of internalization by the developer in malls or by the 
developer and retailer in supermarkets-i.e., in a conversion from streets to malls, $y$ increases from 1 to $\hat{I}_{K}^{m a}$ and in a conversion from streets to supermarkets $y$ increases from 1 to $\hat{I}^{s u}$. We may think of the change taking place in a series of small increases to $y$ at all centers, the effect on $W$ being decomposed as follows:

$$
\frac{d W}{d y}=\left(\frac{\partial W}{\partial y}\right)_{K}+\left(\frac{\partial W}{\partial K}\right)_{v} \frac{d \hat{K}}{d y} .
$$

The first term is the effect of internalization for fixed $K$, which we call the conduct effect, and the second term is the effect of internalization on welfare through the fall in $K$, which we call the $\hat{K}$-reducing effect. ( $\hat{K}$ falls in each of these conversions, by Proposition 6 .) The sign of the $\hat{K}$-reducing effect depends on whether there is too much or too little entry. This is unknown without specifying $T$, but it is well known that excess entry is typical in spatial models (including those used in our examples, namely the Salop model and logit oligopoly).

If there is excess entry then the $\hat{K}$-reducing effect is positive. In a change from streets to supermarkets the conduct effect is nonnegative (Proposition 8) so that the two effects work in the same direction and $W$ increases. Example 4 illustrates this: in a Salop model with vertical $q($ ) there is excess entry, and the supermarket mode increases welfare, even though the conduct effect is zero (because demands are vertical). In a conversion from streets to malls, by contrast, the conduct effect may be negative (Proposition 8), opposing the $\hat{K}$-reducing effect, with ambiguous results for $W$.

In the atypical case of too little entry, the $\hat{K}$-reducing effects are negative. A change from streets to malls or supermarkets here has an ambiguous effect on $W$, as the negative $\hat{K}$-reducing effect may offset any positive conduct effects.

Now consider the practical situation facing a planner who can specify $m$ and limit $K$ below $\hat{K}$, but cannot subsidize unprofitable centers. Suppose the choice is between the street and the supermarket mode. With excess entry, the planner sets $K$ to the socially optimal level, eliminating the $K$-reducing effect. The planner's choice of mode is thus driven entirely by the conduct effect, so the supermarket mode is preferred to the street mode (unless demands are vertical, where mode makes no difference). With insufficient entry, the planner's power to limit $K$ is now irrelevant, and the analysis is as before.

EXAMPLe 4 [Symmetric Salop Circular City]: Linear transportation costs $t$ give $\partial s_{k} / \partial v_{k}=1 / t$. For simplicity, $I_{k}$ and $n_{k}$ are fixed. Retailer costs are $\left\{c_{k}=0, g_{k}=0\right\}$. Developer cost is $f$ per center. Demand is zero-elastic 
at $q_{k}=1$ so a change in $p_{k}$ has no effect on $W$, which is maximized where $K^{w}$ minimizes transportation costs plus fixed costs,

$\arg \min \left\{\frac{t}{4 K}+f K\right\} \Rightarrow K^{w}=\frac{1}{2} \sqrt{t / f}$.

The free-entry condition implies that the profit of the marginal center is zero:

$I_{k} \hat{p}_{K}^{m} / \hat{K}-f=0$.

From the first-order condition (8) it follows that $\hat{p}_{K}^{s t}=s_{k} t=t / K$ and $\hat{p}_{K}^{s u}=s_{k} t / I_{k}=t / I_{k} K$, so (29) gives $\hat{K}^{m}$ :

$\hat{K}^{m}= \begin{cases}\sqrt{I_{k} t / f} & \text { for streets } \\ \sqrt{t / f} & \text { for supermarket. }\end{cases}$

Because $\hat{K}^{s t}>\hat{K}^{s u}>\widehat{K}^{w}$ the planner prefers supermarkets to streets.

\section{CONCLUSIONS}

Over recent decades, across many economies, there has been a striking increase in the extent of internalization of agglomeration effects between product lines at shopping centers. Some of this has occurred at the developer level only, as in the trend from streets to shopping malls. Some has occurred at the level of the developer and the retailer, as in the trend from streets to supermarkets and the rise of retailers such as Wal-Mart that sell a very wide range of products at the same location.

The paper compares the competitive outcomes when shopping centers are organized as streets, malls, and supermarkets. We use a symmetric setting and abstract from all effects other than those resulting from internalization. We allow developers to choose the number of centers and product lines, and allow retailers to set price and quality (or service) levels. For a given number of centers and product lines the results are unambiguous: internalization at retail level results in new price and quality levels, which improve consumer surplus and social efficiency. When the number of product lines is endogenous, as determined by the developer, results depend on the level at which internalization occurs. Developer-only internalization (a change from streets to malls) unambiguously increases the number of product lines but has an ambiguous effect on welfare. In contrast internalization at both levels (a change from streets to supermarkets) has a positive effect on welfare but an ambiguous effect on the number of product lines. Finally, when the number of centers is also endogenous, internalization, whether developer-only, or at the developer and the retailer level, 
reduces the number of centers. Thus, in the case of excess entry, which is typical in spatial models, internalization at retailer and developer level, i.e., a change from streets to supermarkets, unambiguously increases welfare. Overall, therefore, the paper suggests that internalization at the developer and the retailer level, i.e., the change from streets to supermarkets, is favorable from a consumer and social point of view, with more ambiguous results for developer-only internalization.

It is important to note that there are some simplifying assumptions in the analysis. In particular, we have used a symmetric framework, and we assume that product lines are identical and independent in demand and cost. It would be interesting to allow product lines to differ in the externalities they generate, and to allow some competition between retailers in the same center.

\section{Appendix A. Lemmas 1 AND 2}

We define $z^{m}$ as the proportionate rate of change of $s_{k}$ in $v_{k}$ times the retailer's gross per capita profit at optimal prices and quality, i.e.,

$z^{m} \equiv \frac{1}{s_{k}} \frac{\partial s_{k}}{\partial u_{k}} x^{m}\left\{q_{k}\left(\hat{p}_{k}^{m}-c_{k}\right)\right\}=\frac{1}{s_{k}} \frac{\partial \pi_{k}}{\partial s_{k}} \frac{\partial s_{k}}{\partial u_{k}}$

where $x^{m}$ is the retailer's number of product lines in mode $m$. Differentiation of retailer profit $s_{k} x^{m} q_{k}\left(p_{k}-c_{k}\right)$ gives the condition for $\hat{p}_{k}^{m}$ (on any product),

$s_{k} \frac{\partial q_{k}}{\partial p_{k}}\left(\hat{p}_{k}^{m}-c_{k}\right)+q_{k}+q_{k} \frac{\partial s_{k}}{\partial u_{k}} x^{m}\left\{q_{k}\left(\hat{p}_{k}^{m}-c_{k}\right)\right\}=0$.

Substituting from (A1) gives

$s_{k}\left[\frac{\partial q_{k}}{\partial p_{k}}\left(\hat{p}_{k}^{m}-c_{k}\right)+\left(1-z^{m}\right) q_{k}\right]=0$.

This condition implies a limited range for $z^{m}$ as we show in two lemmas.

LEMMA 1: For a profit-maximizing retailer $0<z^{m} \leq 1$.

Proof. Consider the upper limit. Because $q_{k}>0$ at optimal price we maintain $q_{k}>0$ throughout the proof. Thus $z^{m}>1$ implies that the second term in (A2) is negative. Then, for (A2) to hold, the first term must be positive. Because $\partial q_{k} / \partial p_{k} \leq 0$ the first term is positive only if $\hat{p}_{k}^{m}<c_{k}$. However if $\hat{p}_{k}^{m}<c_{k}$ then $z^{m}<0$ by (A1) which contradicts $z^{m}>1$. Thus $z^{m}>1$ is inconsistent with profit maximization. Now consider the lower limit. $z^{m}<0$ implies by (A1) that the retailer makes negative gross profit. This is inconsistent with profit maximization as the retailer could do better by setting $p_{k}=c_{k}$. Now consider $z^{m}=0$. Then the second term 
in (A2) is positive so that the first term in (A2) must be negative. Because $\partial q_{k} / \partial p_{k} \leq 0$ the first term is negative only if $p_{k}>c_{k}$. But $p_{k}>c_{k}$ is inconsistent with $z^{m}=0$ by equation (A1). Thus $z^{m}=0$ cannot satisfy equation (A2). We obtain $0<z^{m} \leq 1$.

LEMMA 2: $z^{m}=1$ if and only if a profit maximizing retailer's products are zero price-elastic at optimal price.

Proof. Beginning with the "if" part, note that if $q()$ is zero price-elastic, the first term in (A2) is zero. Then for (A2) to hold, the second term must be zero. Since $q()>0$ at $\hat{p}_{k}^{m}$, this implies $z^{m}=1$. We now turn to the "only if" part. For (A2) to hold, $z^{m}=1$ implies that the first term is zero for all products. If products are not zero elastic at $\hat{p}_{k}^{m}$ then this requires $\hat{p}_{k}^{m}=c_{k}$ which is inconsistent with $z^{m}=1$ in (A1).

\section{REFERENCES}

Anderson, S.P., A. de Palma, and J.-F. Thisse, 1992, Discrete Choice Theory of Product Differentiation, Cambridge, MA: MIT Press.

Armstrong, M., 2004, "Competition in Two Sided Markets," Working Paper, University College, London.

— and J. Vickers, 2001, "Competitive Price Discrimination," Rand Journal of Economics, 32, 579-605.

Beggs, A.W. 1994, "Mergers and Malls," Journal of Industrial Economics, 44, 419-428.

Bliss, C., 1988, "A Theory of Retail Pricing," Journal of Industrial Economics, 36, 375-391.

Gehrig, T., 1998, "Competing Markets," European Economic Review, 42, 277-310.

Gould, E., P. Pashigian, and C. Prendergast, 2002, “Contracts, Externalities, and Incentives in Shopping Malls," mimeo, University of Chicago.

Mankiw, N.W. and M.D. Whinston, 1986, "Free Entry and Social Efficiency," Rand Journal of Economics, 17, 48-58.

Nocke, V., M. Peitz, and K. Stahl, 2004, “Platform Ownership," Working Paper, University of Pennsylvania.

Pashigian, B. and E. Gould, 1998, “Internalizing Agglomeration Economies: The Pricing of Space in Shopping Malls," Journal of Law and Economics, 41(1), 115-142.

Schulz, N. and K. Stahl, 1996. "Do Consumers Search for the Highest Price? Oligopoly Equilibrium and Monopoly Optimum in Differentiated-Products Markets," Rand Journal of Economics, 27, 542-562.

Shaked, A. and J. Sutton, 1982, “Relaxing Price Competition Through Product Differentiation," Review of Economic Studies, 49, 3-13.

Smith, H.W., 2004 "Price, Quality, and Product Range in Streets, Malls and Supermarkets: Two Examples," mimeo, Department of Economics, University of Oxford.

Spence, M., 1975, "Monopoly Quality and Regulation," Bell Journal of Economics, 6, 417-429.

_ 1976, "Product Selection, Fixed Costs, and Monopolistic Competition," Review of Economic Studies, 43, 271-235.

Spulber, D., 1999, Market Microstructure: Intermediaries and the Theory of the Firm, Cambridge: Cambridge University Press.

Stahl, K., 1982, "Location and Spatial Pricing Theory with Nonconvex Transportation Cost Schedules," Bell Journal of Economics, 13, 575-582.

_ 1987, “Urban Business Location”, in E. S. Mills, ed., Handbook of Regional and Urban Economics, Vol. II, Amsterdam: Elsevier Science Publishers BV. 
